\title{
Effects of Repeated Steroid Injection at Subacromial Bursa With Different Interval
}

\author{
Seung Deuk Byun, $\mathrm{MD}^{1}$, Yong Ho Hong, $\mathrm{MD}^{2}$, Sung Kyung Hong, $\mathrm{MD}^{2}$, Jin Won Song, $\mathrm{MD}^{2}$, \\ Seung Beom Woo, $\mathrm{MD}^{2}$, Jae Hyun Noh, $\mathrm{MD}^{2}$, Jong Min Kim, $\mathrm{MD}^{2}$, Zee Ihn Lee, $\mathrm{MD}^{2}$ \\ ${ }^{1}$ Department of Rehabilitation Medicine, Kang Hospital, Daegu; \\ ${ }^{2}$ Department of Rehabilitation Medicine, Daegu Fatima Hospital, Daegu, Korea
}

Objective To evaluate the effects of repeated steroid injection at subacromial bursa with different interval for patient with periarticular shoulder disorder.

Methods Group A ( $\mathrm{n}=10)$ received subacromial bursa injection only on their first visit, group C ( $\mathrm{n}=10)$ received the injection on their first visit and one week later, and group $B(n=10)$ received the injection on their first visit and two weeks later. All injections were done with a combination of $40 \mathrm{mg}(1.0 \mathrm{~mL})$ of triamcinolone and $5.0 \mathrm{~mL} 0.5 \%$ lidocaine ( $6 \mathrm{~mL}$ total). We examined the active range of motion (AROM) of the shoulder joint, visual analogue scale (VAS), and shoulder disability questionnaire (SDQ) at baseline at 1,2, and 4 weeks after the initial injection. Results In VAS, comparing the changes in VAS between groups, group B showed significant improvements compared with group $A$ or $C$ at 4 weeks after the initial injection $(\mathrm{p}<0.05)$. In SDQ, comparing the changes in SDQ between the groups, group B and C showed more improvement than group A at 4 weeks after the initial injection, but these results were not statistically significant ( $p>0.05$ ). In AROM, comparing the changes in AROM of external rotation between groups, group B and C showed significant improvement compared with group A at 4 weeks after the initial injection $(\mathrm{p}<0.05)$.

Conclusion It may be more effective in pain relief for patients with periarticular disorder to receive subacromial bursa injections twice with 2-week interval, as opposed to once.

Keywords Effect repeated steroid injection, Subacromial bursa, Periarticular shoulder disorder

Received April 3, 2014; Accepted July 29, 2014

Corresponding author: Yong Ho Hong

Department of Rehabilitation Medicine, Daegu Fatima Hospital, 99 Ayang-ro, Dong-gu, Daegu 701-724, Korea

Tel: +82-53-940-7824, Fax: +82-53-954-7417, E-mail: hyh8206@nate.com

(c) This is an open-access article distributed under the terms of the Creative Commons Attribution Non-Commercial License (http://creativecommons. org/licenses/by-nc/3.0) which permits unrestricted noncommercial use, distribution, and reproduction in any medium, provided the original work is properly cited.

Copyright $\odot 2014$ by Korean Academy of Rehabilitation Medicine

\section{INTRODUCTION}

The injection at the subacromial bursa is a widely used method of treatment to alleviate the pain and to improve the function of patients with periarticular shoulder disorder, and the most widely used type of injection is steroid and lidocaine [1-4]. Yoon et al. [1] reported that the group that received steroid injection for the shoulder disease showed significant improvement with pain control, shoulder joint motion, and function. While there is a study for the effect of steroid injection according to dosage and there is an animal study for the adverse effect of 
steroid injection according to the number of injections. There are not enough studies on the appropriate number and interval of steroid injection in the bursa and there is no generally accepted protocol $[5,6]$. With greater dose and frequency of steroid injection, the steroid increases degenerative changes and causes metabolic and endocrinological side effects $[2,7]$, it is deemed that there should be standards on the appropriate number and interval. The authors have administered steroid injection at the subacromial bursa with different numbers and intervals in the patients with periarticular shoulder disorder and compared the effect.

\section{MATERIALS AND METHODS}

\section{Subjects}

The subjects were the patients who visited the Department of Rehabilitation Medicine at Daegu Fatima Hospital for shoulder pain between March 2012 and September 2012. Among them, we selected the cases that clinically had painful arc, were positive to the Hawkins test or Neer impingement sign, and showed partial or full thickness tear of the rotator cuff or subacromial bursitis in the ultrasonography. We included patients with limited range of active joint motion due to pain. Exclusions included 1) cases with suspected adhesive capsulitis with limited range of passive joint motion, 2) cases with history of shoulder surgery, 3) cases with history of steroid or hyaluronic injection at the shoulder due to the same cause, 4) cases with hemiplegic shoulder pain, and 5) cases with suspected fracture based on the simple X-ray. Qualified patients were introduced to the methodology and purpose of study and those who gave consent were randomly assigned to groups A, B, and C, each of which had 10 members.

\section{Injection in subacromial bursa}

After the physical examination and the assessment of shoulder pain and function, the lesion was examined by one physiatrist using ultrasonography. Then, the patient's arm was internally rotated and extended to attach the lower arm to the patients back in the seated position. The injection site was aseptically sterilized to have the same specialist administer the injection at the subacromial bursa under ultrasonographic guide. All patients in each group received $40 \mathrm{mg}(1.0 \mathrm{~mL})$ of triamcinolone acetonide (Dongkwang Pharm., Seoul, Korea) and 5.0 $\mathrm{mL}$ 0.5\% lidocaine; the injection was administered once for group A, twice with 2-week interval for group B, and twice with 1-week interval for group C. The patients were asked to stop taking any pain medication or anti-inflammatory drugs from one week in prior to the injection, and they were trained to perform shoulder muscle stabilization exercises and shoulder extension exercises at home after the injection. They were trained and recommended to perform shoulder muscle stabilization exercise and other exercises during every follow-up examination. No additional physical therapies or medications were performed.

\section{Assessment of effect of injection}

The patients were assessed four times for comparison, once before the first injection and at 1,2, and 4 weeks after the injection. The assessment was performed by the same assessor who was in charge of assessment before the injection. The average value of degree of pain for the previous 24 hours was asked and measured on the visual analogue scale (VAS, 0 to 10). The elbow joint was extended in the seated position to measure the range of shoulder flexion and abduction at the sagittal and coronal planes. The range of external rotation and internal rotation was measured with the shoulder joint abducted by $90^{\circ}$ and the elbow joint flexed by $90^{\circ}$ in the seated position. The normal range of flexion and abduction of shoulder joint was $180^{\circ}$, and the normal range of external rotation and internal rotation was $90^{\circ}$. The shoulder disability questionnaire (SDQ) was used to assess the shoulder function of the patients. SDQ is an assessment tool with proven feasibility and reliability and assesses on the scale of 0 (no disorder) through 22 (maximum disorder), and the degree of shoulder disorder is perceived by each patient with 22 self-report questions (Appendix 1).

\section{Statistical analysis}

The statistical analysis used SPSS ver. 12.0 for the Windows (SPSS Inc., Chicago, IL, USA). The measurements were taken four times before the injection and at 1,2 , and 4 weeks after the injection were assessed using the Wilcoxon singed-rank test and the Mann-Whitney U test, which was used to compare the degree of change in the measurements for each groups, and the ANOVA was used to compare the degree of change in the measurements 
for all three groups. It was analyzed that the change was statistically significant when the p-value was below 0.05 .

\section{RESULTS}

Demographics and ultrasonographic findings of the subjects

The three groups did not show any significant difference in sex, age, and place of lesion before the injection, and there was no statistically significant difference in each scale before the injection (Table 1). The three groups' ultrasonographic diagnoses were performed before the injection and did not show any significant difference (Table 1).

Table 1. General characteristics of subjects and initial ultrasonographic diagnosis

\begin{tabular}{lccc}
\hline & $\begin{array}{c}\text { Group A } \\
(\mathbf{n}=10)\end{array}$ & $\begin{array}{c}\text { Group B } \\
(\mathbf{n}=\mathbf{1 0})\end{array}$ & $\begin{array}{c}\text { Group C } \\
(\mathbf{n}=\mathbf{1 0})\end{array}$ \\
\hline Sex (male:female) & $5: 5$ & $4: 6$ & $5: 5$ \\
Age (yr) & $63.3 \pm 10.0$ & $63.9 \pm 8.0$ & $60.2 \pm 7.9$ \\
Shoulder affected & & & \\
$\quad$ Dominant & $6(60.0)$ & $7(70.0)$ & $7(70.0)$ \\
\hline Non-dominant & $4(40.0)$ & $3(30.0)$ & $3(30.0)$ \\
VAS & $5.8 \pm 1.3$ & $5.7 \pm 1.3$ & $5.1 \pm 0.9$ \\
SDQ & $8.8 \pm 5.4$ & $9.9 \pm 4.8$ & $9.7 \pm 4.5$ \\
ROM $\left(^{\circ}\right)$ & & & \\
\hline Flexion & $163.0 \pm 6.7$ & $165.0 \pm 12.7$ & $167.0 \pm 9.5$ \\
\hline Abduction & $158.0 \pm 15.5$ & $153.0 \pm 30.2$ & $160.0 \pm 14.9$ \\
\hline IR & $70.0 \pm 15.6$ & $75.0 \pm 19.0$ & $77.0 \pm 11.6$ \\
\hline ER & $82.0 \pm 9.2$ & $79.0 \pm 8.8$ & $74.0 \pm 14.3$
\end{tabular}

Ultrasonographic diagnosis

\begin{tabular}{llll} 
RC partial tear & 3 & 4 & 4 \\
RC full-thickness tear & 4 & 2 & 3 \\
SA-SD bursitis & 3 & 4 & 3 \\
\hline
\end{tabular}

Values are presented as mean \pm standard deviation or number (\%).

Group A, underwent subacromial bursa injection only at the 1st visit; group B, underwent subacromial bursa injection at the 1st visit and 2 weeks later (with two weeks interval); group C, underwent subacromial bursa injection at the 1st visit and 1 week later (with one week interval); VAS, visual analogue scale; SDQ, shoulder disability questionnaire; ROM, range of motion; IR, internal rotation; ER, external rotation; RC, rotator cuff; SA-SD, subacromial-subdeltoid.

\section{Change in VAS of pain}

All three groups showed statistically significant improvement when we compared their VAS from before the first injection and at 1,2, and 4 weeks after the injection (Table 2). Group B particularly showed statistically significant improvement compared with group A and group $\mathrm{C}$ when the measurements from before the injection were compared with those from 4 weeks after the injection (Table 3).

\section{Change in SDQ}

All three groups showed statistically significant improvement when SDQ was compared from before the first injection and at 1,2, and 4 weeks after the injection (Table 2). Groups B and C showed greater improvement compared with group A when the measurements from before the injection were compared with those from 4 weeks after the injection, but it was not statistically significant (Table 3).

\section{Changes in active range of motion}

All three groups showed statistically significant improvement in flexion and external rotation when measurements from before the injection were compared with those from 1, 2, and 4 weeks after the injection (Table 2). Groups B and C showed statistically significantly greater improvement compared with group A in external rotation when the measurements from before the injection were compared with those from 4 weeks after the injection (Table 3).

\section{DISCUSSION}

Steroid injection at the subacromial bursa is widely used to reduce pain and improve function in the periarticular shoulder disorder. Arroll and Goodyear-Smith [8] performed meta-analysis of a report in which corticosteroid effectively restored the range of motion and reduced pain compared with a placebo or non-steroidal anti-inflammatory drugs. Tillander et al. [9] examined histological changes of rotator cuffs when triamcinolone was repetitively injected at the subacromial bursa of normal rats three times with 1-week interval and when it was repetitively injected eight times, reporting that the rotator cuffs showed no histological change after three injections, whereas necrosis and partial inflammation 
Table 2. Summary of treatment effects in group A, B, and C

\begin{tabular}{|c|c|c|c|c|c|}
\hline & Group & Pre-injection & 1 week after lst injection & 2 weeks after 1 st injection & 4 weeks after 1st injection \\
\hline \multirow[t]{3}{*}{ VAS } & $\mathrm{A}$ & $5.8 \pm 1.3$ & $3.0 \pm 1.4^{*}$ & $2.4 \pm 1.2^{*}$ & $2.4 \pm 1.5^{*}$ \\
\hline & B & $5.7 \pm 1.3$ & $3.5 \pm 1.8^{*}$ & $2.5 \pm 1.6^{*}$ & $0.7 \pm 0.8^{*}$ \\
\hline & $\mathrm{C}$ & $5.1 \pm 0.9$ & $3.1 \pm 1.2^{*}$ & $1.4 \pm 0.8^{*}$ & $1.5 \pm 0.7^{*}$ \\
\hline \multirow[t]{3}{*}{ SDQ } & A & $8.8 \pm 5.4$ & $3.7 \pm 3.7^{*}$ & $3.7 \pm 3.7^{*}$ & $3.8 \pm 5.6^{*}$ \\
\hline & B & $9.9 \pm 4.8$ & $6.3 \pm 4.2^{*}$ & $5.0 \pm 3.9 *$ & $2.1 \pm 2.0^{*}$ \\
\hline & $\mathrm{C}$ & $9.7 \pm 4.5$ & $7.2 \pm 3.7^{*}$ & $2.5 \pm 1.9^{*}$ & $2.1 \pm 1.9 *$ \\
\hline \multicolumn{6}{|l|}{$\operatorname{ROM}\left({ }^{\circ}\right)$} \\
\hline \multirow[t]{3}{*}{ Flexion } & A & $163.0 \pm 6.7$ & $170.0 \pm 6.7^{*}$ & $171.0 \pm 8.8^{*}$ & $173.0 \pm 6.7^{*}$ \\
\hline & B & $165.0 \pm 12.7$ & $171.5 \pm 11.6^{*}$ & $174.0 \pm 8.4^{*}$ & $178.0 \pm 4.2^{*}$ \\
\hline & $\mathrm{C}$ & $167.0 \pm 9.5$ & $176.0 \pm 6.7^{*}$ & $179.0 \pm 3.2^{*}$ & $179.0 \pm 3.2^{*}$ \\
\hline \multirow[t]{3}{*}{ Abduction } & A & $158.0 \pm 15.5$ & $170.0 \pm 6.7^{*}$ & $173.0 \pm 6.7^{*}$ & $172.0 \pm 7.8^{*}$ \\
\hline & B & $153.0 \pm 30.2$ & $164.0 \pm 21.3^{*}$ & $170.5 \pm 13.0^{*}$ & $177.5 \pm 4.2^{*}$ \\
\hline & $\mathrm{C}$ & $160.0 \pm 14.9$ & $175.0 \pm 9.7^{*}$ & $178.0 \pm 6.3^{*}$ & $178.0 \pm 6.3^{*}$ \\
\hline \multirow[t]{3}{*}{ IR } & A & $70.0 \pm 15.6$ & $79.0 \pm 11.9^{*}$ & $81.0 \pm 12.6^{*}$ & $80.0 \pm 13.3^{*}$ \\
\hline & B & $75.0 \pm 19.0$ & $79.0 \pm 14.5$ & $83.0 \pm 8.2$ & $84.0 \pm 9.7$ \\
\hline & $\mathrm{C}$ & $77.0 \pm 11.6$ & $85.0 \pm 7.1^{*}$ & $89.0 \pm 3.2^{*}$ & $89.0 \pm 3.2^{*}$ \\
\hline \multirow[t]{3}{*}{ ER } & A & $82.0 \pm 9.2$ & $85.0 \pm 8.5$ & $86.0 \pm 6.6$ & $84.0 \pm 8.1$ \\
\hline & B & $79.0 \pm 8.8$ & $85.0 \pm 7.1^{*}$ & $88.0 \pm 4.2^{*}$ & $90.0 \pm 0.0^{*}$ \\
\hline & $\mathrm{C}$ & $74.0 \pm 14.3$ & $88.0 \pm 4.2^{*}$ & $89.5 \pm 1.6^{*}$ & $88.5 \pm 3.4^{*}$ \\
\hline
\end{tabular}

Values are presented as mean \pm standard deviation.

Group A, underwent subacromial bursa injection only at the 1st visit; group B, underwent subacromial bursa injection at the 1st visit and 2 weeks later (with two weeks interval); group C, underwent subacromial bursa injection at the 1 st visit and 1 week later (with one week interval); VAS, visual analogue scale; SDQ, shoulder disability questionnaire; ROM, range of motion; IR, internal rotation; ER, external rotation.

${ }^{*} \mathrm{p}<0.05$ for comparison of the pre-injection with the after injection by the Wilcoxon signed-rank test.

were observed after eight injections. The effect of steroid injection at subacromial bursa has been proven, but it is important that administering it at the appropriate frequency and interval because there may be degenerative change or metabolic and endocrinological side effects as the dosage of steroid increases with repeated administration $[1,7]$. As there is no generally accepted protocol, the authors have administered steroid injection at the subacromial bursa in the patients with periarticular shoulder disorder while varying the number and interval in order to compare the effect. Hong et al. [10] examined the groups that received the injection of $40 \mathrm{mg}$ or $20 \mathrm{mg}$ triamcinolone and placebo at subacromial bursa for the periarticular shoulder disorder in weeks 2, 4, and 8 and reported that the groups with $40 \mathrm{mg}$ or $20 \mathrm{mg}$ triamcinolone showed effective changes in VAS, SDQ, and active range of motion compared with a placebo group. They particularly reported that $40 \mathrm{mg}$ triamcinolone was more effective than $20 \mathrm{mg}$ triamcinolone. In the assessment of effect, both of these groups with $40 \mathrm{mg}$ or $20 \mathrm{mg}$ triamcinolone showed significant improvements after 2, 4, and 8 weeks, but the change was the most after 4 weeks. Therefore, this study compared the measurements from before the injection to those from 4 weeks after the injection to assess the effect of treatment.

The change in SDQ improved with statistical significance in all groups when the measurements from before the injection were compared with those from 1,2, and 4 weeks after the injection. In terms of the change in active range of motion, all three groups showed statistically significant improvement in flexion and external rotation when the measurements from before the injection were compared with those from 1,2, and 4 weeks after the injection. When the improvement was compared, external rotation showed statistical significant improvement in the groups where the injection was administered 
Table 3. Changes in measurement values of group A, B, and $\mathrm{C}$

\begin{tabular}{lccc}
\hline & Group A & Group B & Group C \\
\hline VAS & $3.5 \pm 1.6$ & $5.1 \pm 1.3^{\text {tै }}$ & $3.6 \pm 1.1$ \\
SDQ & $5.0 \pm 3.2$ & $7.8 \pm 3.1$ & $7.6 \pm 3.9$ \\
ROM $\left(^{\circ}\right)$ & & & \\
\hline Flexion & $10.0 \pm 6.7$ & $13.0 \pm 10.6$ & $12.0 \pm 7.9$ \\
\hline Abduction & $14.0 \pm 12.6$ & $24.5 \pm 28.1$ & $18.0 \pm 11.4$ \\
\hline IR & $10.0 \pm 8.2$ & $9.0 \pm 10.7$ & $12.0 \pm 10.3$ \\
\hline ER & $2.0 \pm 8.9$ & $11.0 \pm 8.8^{\dagger}$ & $14.5 \pm 14.2^{*}$ \\
\hline
\end{tabular}

Values are presented as mean \pm standard deviation.

Group A, underwent subacromial bursa injection only at the 1st visit; group B, underwent subacromial bursa injection at the 1 st visit and 2 weeks later (with two weeks interval); group $\mathrm{C}$, underwent subacromial bursa injection at the 1st visit and 1 week later (with one week interval); VAS, visual analogue scale; SDQ, shoulder disability questionnaire; ROM, range of motion; IR, internal rotation; ER, external rotation.

${ }^{*} \mathrm{p}<0.05$ for comparison of the group A with the group $\mathrm{C}$ by the Mann-Whitney U test.

${ }^{\dagger} \mathrm{p}<0.05$ for comparison of the group A with the group B by the Mann-Whitney U test.

${ }^{*} \mathrm{p}<0.05$ for comparison of the group B with the group C by the Mann-Whitney U test.

twice with 1-week interval and twice with 2-week interval compared with the group where the injection was administered once. In the change of VAS, all groups showed statistical significant improvement when VAS was compared from before the injection and 1, 2, and 4 weeks after the injection. When the improvement was compared from before the injection and 4 weeks after the injection, the group, where the injection was administered twice with 2-week interval, showed more statistical significant improvement compared with the groups where the injection was administered once and twice with 1-week interval. Considering these findings, it was concluded that administering the subacromial steroid injection twice with 2-week interval would be effective for reducing the pain for the patients. As the subjects of this study were the patients with periarticular shoulder disorder, the patients with adhesive capsulitis were excluded from the selection to avoid the patients with capsular pattern. In case of early adhesive capsulitis, it is difficult to tell the difference from the shoulder impingement syndrome as there could be no definite limitation of passive range of motion so it could be a limitation of this study.
Triamcinolone is an intermediate acting steroid and it is known that it stays effective for about 2 weeks; but, without the generally accepted protocol, the patients are mostly followed up every week for the repeat injection. In this study, when groups B and C were compared before the injection and 4 weeks after the injection, the average VAS of group B before the injection was $5.7 \pm 1.3$ and that of group C was $5.1 \pm 0.9$. The average VAS of group B after 4 weeks was $0.7 \pm 0.8$ and that of group $C$ was $1.5 \pm 0.7$. The groups' change in VAS from before the injection and 4 weeks after the injection was $5.1 \pm 1.3$ for group B and $3.6 \pm 1.1$ for group C. VAS decreased significantly for both groups B and C, but group B's VAS decreased with more statistical significance than group C's VAS. Also, group B's average VAS was lower than group C's average VAS, solely considering VAS from week 4; but, the better results of group B were possible because the effect still remained. Considering that the steroid generally stays effective for more than 2 weeks, administering steroid injection with 1 -week interval would create the overlapping period. If the purpose of treatment is to extend the effect, therefore, 2-week injection interval would be more effective than one-week injection interval. Also, the risk of side effects would be greater due to excessive steroid dosage during the overlapping period. It is a limitation that this study had a limited number of subjects and failed to examine the long-term effect of steroid injection after 4 weeks of injection as the follow-up examination was lost when the symptoms improved after the injection. Additional studies would be necessary with longer follow-up periods with a great number of subjects. These studies would suggest the generally accepted protocol that is most effective with fewer side effects of steroid.

In conclusion, it may be more effective to reduce the pain for the patients with periarticular shoulder disorder to administer $40 \mathrm{mg}$ steroid injection at the subacromial bursa twice with 2-week interval as opposed to administering the injection once.

\section{CONFLICT OF INTREST}

No potential conflict of interest relevant to this article was reported. 


\section{REFERENCES}

1. Yoon SH, Kwack KS, Rah UW, Cho KH. Ultrasonography-guided subacromial bursal injection of corticosteroid: a comparative study of two dose regimens. J Korean Acad Rehabil Med 2009;33:402-7.

2. Naredo E, Cabero F, Beneyto P, Cruz A, Mondejar B, Uson J, et al. A randomized comparative study of short term response to blind injection versus sonographicguided injection of local corticosteroids in patients with painful shoulder. J Rheumatol 2004;31:308-14.

3. Chen MJ, Lew HL, Hsu TC, Tsai WC, Lin WC, Tang SF, et al. Ultrasound-guided shoulder injections in the treatment of subacromial bursitis. Am J Phys Med Rehabil 2006;85:31-5.

4. Blair B, Rokito AS, Cuomo F, Jarolem K, Zuckerman JD. Efficacy of injections of corticosteroids for subacromial impingement syndrome. J Bone Joint Surg Am 1996;78:1685-9.

5. Akpinar S, Hersekli MA, Demirors H, Tandogan RN, Kayaselcuk F. Effects of methylprednisolone and betamethasone injections on the rotator cuff: an experi- mental study in rats. Adv Ther 2002;19:194-201.

6. Doumouchtsis SK, Boama V, Gorti M, Tosson S, Fynes MM. Prospective evaluation of combined local bupivacaine and steroid injections for the management of chronic vaginal and perineal pain. Arch Gynecol Obstet 2011;284:681-5.

7. Byun SD, Park DH, Jo DH, Choi WD, Lee ZI. Effect of three consecutive steroid injection on blood glucose and cortisol level in diabetic patients with hemiplegic shoulder pain. Clin Pain 2010;9:82-9.

8. Arroll B, Goodyear-Smith F. Corticosteroid injections for painful shoulder: a meta-analysis. Br J Gen Pract 2005;55:224-8.

9. Tillander B, Franzen LE, Karlsson MH, Norlin R. Effect of steroid injections on the rotator cuff: an experimental study in rats. J Shoulder Elbow Surg 1999;8:271-4.

10. Hong JY, Yoon SH, Moon DJ, Kwack KS, Joen B, Lee HY. Comparison of high- and low-dose corticosteroid in subacromial injection for periarticular shoulder disorder: a randomized, triple-blind, placebo-controlled trial. Arch Phys Med Rehabil 2011;92:1951-60. 
Appendix 1. Shoulder Disability Questionnaire (Good: 0, Bad: 22)

1. It is difficult to move my arm and hand because my shoulder hurts.

2. I cannot take a bath by myself because my shoulder hurts.

$\square$ Yes

No

3. Someone has to help me put on the clothes because of my shoulder.

Yes

No

4. I have to put on the clothes slowly because my shoulder hurts.

Yes

No

5. I have difficulties closing my clothes because of my shoulder (buttons, zipper, etc).

Yes

No

6. I have difficulties putting on my coat, shirt, blouse, or jacket because of my shoulder.

Yes

No

7. I have to turn often when I sleep because my shoulder hurts.

$\square$ Yes $\quad \square$ No

8. I cannot sleep on my right side because my shoulder hurts.

Yes

No

9. I cannot sleep on my left side because my shoulder hurts.

Yes

No

10. I spend most of my time at home because my shoulder hurts.

Yes

No

11. The amount of house chores I can do has decreased because my shoulder hurts.

$\square$ Yes

$\square$ No

12. I avoid arduous tasks around the home because my shoulder hurts.

Yes

No

13. I cannot lift up things when shopping because my shoulder hurts.

Yes

No

14. I had to play sports or do leisure activities less because of my shoulder.

Yes

No

15. I cannot engage in recreational or leisure activities because of my shoulder.

Yes

No

16. I try to find someone to help me because my shoulder hurts.

Yes

No

17. I tend to get upset or irritated more often because of my shoulder.

Yes

No

18. I cause minor accidents more often because of my shoulder (dropping things).

Yes

No

19. I can hardly fall asleep because of my shoulder.

Yes

No

20. I try to rest more because of my shoulder.

Yes

$\square$ No

21. I have poor appetite because of my shoulder.

Yes

No

Yes

$\square$ No

22. I have difficulties writing or typing because of my shoulder.

Yes

No 